\title{
MODELING THE OXYGEN K ABSORPTION IN THE INTERSTELLAR MEDIUM: AN XMM-NEWTON VIEW OF Sco X-1
}

\author{
J. García ${ }^{1,2}$, J. M. Ramírez ${ }^{3}$, T. R. Kallman ${ }^{2}$, M. Witthoeft ${ }^{2}$, M. A. Bautista ${ }^{1}$, \\ C. MendozA ${ }^{4}$, P. PAlmeri ${ }^{5}$, And P. Quinet ${ }^{5,6}$ \\ ${ }^{1}$ Department of Physics, Western Michigan University, Kalamazoo, MI 49008, USA; javier.garcia@wmich.edu, manuel.bautista@wmich.edu \\ 2 NASA Goddard Space Flight Center, Greenbelt, MD 20771, USA; timothy.r.kallman@nasa.gov, michael.c.witthoeft@nasa.gov \\ ${ }^{3}$ Astrophysikalisches Institut Potsdam, An der Sternwarte 16 D-14482 Potsdam, Germany; jramirez@aip.de \\ ${ }^{4}$ Centro de Física, IVIC, Caracas 1020, Venezuela; claudio@ivic.gob.ve \\ ${ }^{5}$ Astrophysique et Spectroscopie, Université de Mons-UMONS, B-7000 Mons, Belgium; palmeri@umh.ac.be \\ ${ }^{6}$ IPNAS, Sart Tilman B15, Université de Liège, B-4000 Liège, Belgium; quinet@umh.ac.be \\ Received 2011 January 5; accepted 2011 February 8; published 2011 March 21
}

\begin{abstract}
We investigate the X-ray absorption structure of oxygen in the interstellar medium by analyzing XMM-Newton observations of the low-mass X-ray binary Sco X-1. Simple models based on the O I atomic photoabsorption cross section from different sources are used to fit the data and evaluate the impact of the atomic data on the interpretation of the observations. We show that relatively small differences in the atomic calculations can yield spurious results, and that the most complete and accurate set of atomic cross sections successfully reproduce the observed data in the 21.0-24.5 $\AA$ wavelength region of the spectrum. Our fits indicate that the absorption is mainly due to neutral gas with an ionization parameter of $\xi=10^{-4} \mathrm{erg} \mathrm{cm} \mathrm{s}^{-1}$ and an oxygen column density of $N_{\mathrm{O}} \approx(8-10) \times 10^{17} \mathrm{~cm}^{-2}$. The models are able to reproduce both the $\mathrm{K}$ edge and the $\mathrm{K} \alpha$ absorption line from $\mathrm{O}$ I which are the two main features in this region. We find no conclusive evidence for absorption by anything other than atomic oxygen.
\end{abstract}

Key words: atomic data - atomic processes - dust, extinction - line: formation - X-rays: general

\section{INTRODUCTION}

X-ray spectroscopy provides a powerful tool for understanding the physical and chemical properties of the diffuse interstellar medium (ISM). The X-ray band covers the emission and absorption spectra produced by inner-shell transitions of the most abundant ions from carbon to iron. The interaction of $\mathrm{X}$-ray photons from bright point sources (e.g., galactic X-ray binaries) with the ISM gives rise to absorption lines and edges in the spectrum. The energy position and the shape of these features depend on whether the absorption is due to free atoms or molecules, and on whether these atoms or molecules are in the gas or in the solid phase.

Neutral oxygen is a major constituent of the ISM which makes it one of the most important elements in astronomical observations. Precise knowledge of the neutral oxygen atomic properties is needed for the correct modeling of the observed spectra. Calculations of the photoabsorption cross section of the ground state of O I were carried out by McLaughlin \& Kirby (1998, hereafter мск98) using the $R$-matrix method, giving a detailed comparison with the experimental results of Stolte et al. (1997). Although they claimed overall agreement, there are significant discrepancies in the positions of the inner-shell excited resonances and in the near-threshold resonance profiles. This problem was overcome in the $L S$-coupling calculation of Gorczyca \& McLaughlin (2000, hereafter GMC00) by taking into account core relaxation effects and the smearing of the $\mathrm{K}$ edge due to Auger damping. A more complete $R$-matrix calculation was carried out in intermediate coupling by García et al. (2005, hereafter GAR05) for all the ions in the oxygen isonuclear sequence. There is now very good agreement between the GAR05 calculations and both the experimental cross section (Stolte et al. 1997) and the GMC00 results.

The oxygen inner-shell features in the X-ray spectrum of galactic sources have been used to provide abundance determi- nations in the ISM as well as estimates of the oxygen ionization fractions (Schulz et al. 2002; Takei et al. 2002; Juett et al. 2004, 2006; Turner et al. 2004; Ueda et al. 2005). However, studies in the IR and UV have shown that oxygen can also be found in solid particles (Draine 2003; Whittet 2003). It has been argued that oscillatory modulations near the $\mathrm{K}$ edge, usually referred to as the X-ray absorption fine structure (XAFS), could be detected. These are condensed matter modulations of the atomic cross section due to the presence of solid particles (Lee \& Ravel 2005; Lee et al. 2009). Studies of the soft X-rays from galactic sources have reported possible detections of molecules that could be linked to XAFS signatures in the edges of several elements such as $\mathrm{Ne}, \mathrm{Si}$, and $\mathrm{Mg}$ (Paerels et al. 2001; Lee et al. 2002; Ueda et al. 2005), in the L edge of Fe (Lee et al. 2001; Kaastra et al. 2009), and in particular, in the oxygen K edge (De Villiers et al. 2003; Costantini et al. 2005; de Vries \& Costantini 2009; Pinto et al. 2010). Although these signatures could be important in the $\mathrm{K}$ edges of molecules involving higher $Z$ elements, namely, $\mathrm{Mg}, \mathrm{Si}$, and $\mathrm{Fe}$, oxygen is $\sim 10-20$ times more abundant than any of these, thus potentially providing enough signal-to-noise to detect the XAFS signatures. Theoretical models of XAFS in the astrophysical context have been developed by Woo (1995), Woo et al. (1997), and Forrey et al. (1998). See also Lee (2011) and references therein for details on the theory of XAFS.

$X M M-N e w t o n$ observations of the X-ray source Scorpius $\mathrm{X}-1$ (Sco X-1) reveal strong absorption in the wavelength region corresponding to neutral oxygen. Located at a $\sim 2.8 \mathrm{kpc}$ distance (Bradshaw et al. 1999) and with a flux of $F \sim 3.4 \times$ $10^{8} \mathrm{erg} \mathrm{cm}^{-2} \mathrm{~s}^{-1}$ (in the 2-10 keV energy band), it is the brightest X-ray source in the sky other than the Sun and the diffuse $\mathrm{X}$-ray background radiation. Its high $\mathrm{X}$-ray flux provides very good statistics in relatively short exposure times, giving the opportunity to study signatures of oxygen absorption in the ISM with great detail. For several galactic sources, including 
Sco X-1, de Vries et al. (2003) analyzed high-resolution X-ray spectra taken with the reflection grating spectrometer (RGS) in the XMM-Newton satellite. By comparing low- and highextinction sources, they were able to separate the ISM and the instrumental components of the O I K edge; moreover, de Vries \& Costantini (2009) searched for XAFS signatures in the spectrum of Sco X-1. The XAFS signature is derived from the differences between the observed flux and that predicted theoretically. However, the model used by these authors is based on the atomic oxygen absorption cross section calculated by мСК 98 .

In this Letter, we show the importance of the accuracy of the atomic data used in the modeling of the detailed features of the oxygen absorption in the ISM. We demonstrate that small variations in the K-edge structure derived from different atomic calculations yield spurious results when applied to astronomical observations. In Section 2, we describe the observational data used in our study while the theoretical models are delineated in Section 3. Results derived from model fits of the observed data are presented in Section 4, and finally, the main conclusions are summarized in Section 5.

\section{OBSERVATION AND DATA REDUCTION}

For the purpose of this work, we adopt the XMM-Newton spectrum of Sco X-1 taken in orbit $0592($ ObsID = 0152890101) with the RGS instrument (den Herder et al. 2001). The general observational strategy is described in detail in de Vries et al. (2003) and de Vries \& Costantini (2009) and will not be given here. Taking into account the high X-ray flux of Sco X-1, standard spectroscopy mode would lead to a very high pileup level. This was avoided by choosing a faster readout mode which is able to read each of the nine RGS CCDs in separate exposures. Because of a failure in the RGS2, we make use of only the RGS1 data in what follows. During this observation there were 24 separate contiguous exposures, but only the fifth and the eighteenth contained calibrated and reliable data in the 21-25 A spectral range; thus, we only use these exposures in our spectral analysis. In the notation of the SAS rgsproc task, these two exposures are labeled S005 and S018. We follow the standard procedure for the reduction and extraction of the RGS spectrum using SAS version 10 with the latest calibration files (CCFs). We finally present the spectrum with the default binning of $0.05 \AA$ A employing full spectrometer resolution.

\section{SPECTROSCOPIC MODELS}

\subsection{Atomic Data}

Figure 1 shows the photoabsorption cross section of the ground state of neutral oxygen in the 22.5-24.0 $\AA$ wavelength region from the calculations by MCK98, GMC00, and GAR05. All these curves have been convoluted with a Gaussian profile of $0.182 \mathrm{eV}$ full width at half-maximum (FWHM) in order to match the resolution of those presented in GMC00. This spectral region covers both the $\mathrm{K}$ edge around $22.5 \AA$ and the $\mathrm{K} \alpha 1 s-2 p$ absorption line near $23.5 \AA$. Besides the differences in the $\mathrm{K} \alpha$ position, the GAR05 and GMC00 calculations agree very well. Nevertheless, there are significant discrepancies with MCK98, particularly in the shape of the $\mathrm{K}$ edge and in the energy separation between the $\mathrm{K}$ edge and the $\mathrm{K} \alpha$ resonance. Since the $\mathrm{K} \alpha$ absorption feature is prominent and well resolved and its energy is experimentally fixed, it is used as a reference in most spectral fits. Thus, uncertainties in the energy and shape of the cross section around the inner-shell edges are translated in the form of spurious residuals in the fits.

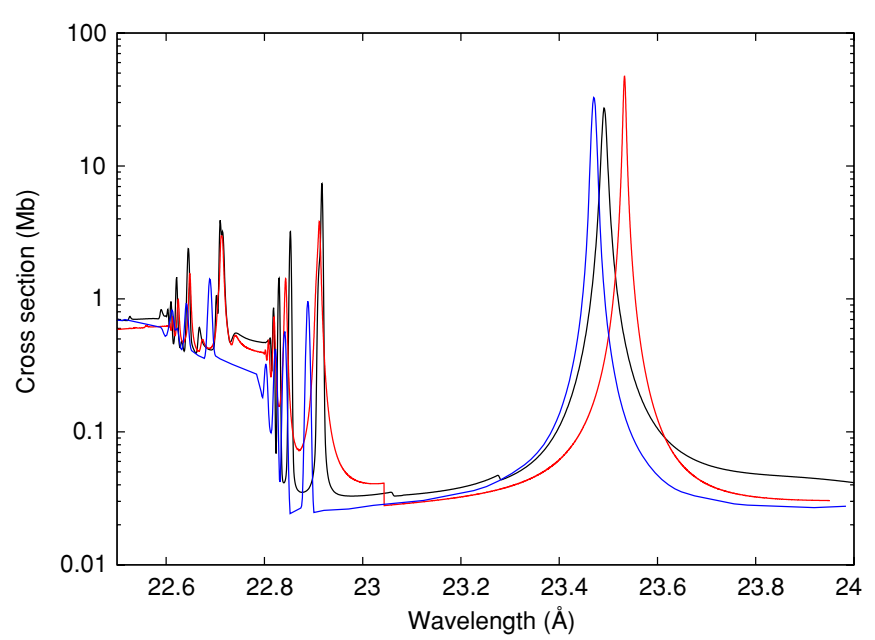

Figure 1. Comparison of the theoretical photoabsorption cross sections of neutral oxygen in the 22.5-24.0 $\AA$ wavelength region: McLaughlin \& Kirby (1998, blue curve), Gorczyca \& McLaughlin (2000, red curve), and García et al. (2005, black curve). This spectral region covers both the absorption K edge and the $\mathrm{K} \alpha$ absorption line $(1 s-2 p)$ from $\mathrm{O}$ I. All the curves have been convoluted with a $0.182 \mathrm{eV}$ FWHM Gaussian profile.

These discrepancies have been formally addressed by GMC00 as the result to two conspicuous effects: orbital relaxation due to the $\mathrm{K}$ shell vacancy which affects the atomic structure and, consequently, the resonance and edge positions; and resonance decay through an infinite number of transitions wherein the resonance width is usually dominated by the spectator-Auger channel. It is evident from the comparison in Figure 1 that the neglect of these two effects underestimates the absorption cross section near the K edge by $20 \%-50 \%$.

\subsection{Model A}

In order to resolve the features in the Sco X-1 observation, we have employed the photoionization code XSTAR. Several calculations were performed covering a range of parameters, the most important being the hydrogen column density, $N_{\mathrm{H}}$, and the ionization parameter, $\xi=L / n R^{2}$, where $L$ is the luminosity of the source, $R$ its distance, and $n$ is the density of the gas (Tarter et al. 1969). We have constructed a grid of XSTAR models spanning hydrogen column densities of $N_{\mathrm{H}}=10^{19}-10^{22} \mathrm{~cm}^{-2}$ and ionization parameters of $\xi=10^{-4}-10 \mathrm{erg} \mathrm{cm} \mathrm{s}^{-1}$ with the gas density fixed at $n=10^{3} \mathrm{~cm}^{-3}$. The spectral region of interest, $21.0-24.5 \AA$, is relatively small and, in practice, is dominated by oxygen species. Thus, the XSTAR models include hydrogen, helium, and oxygen ions in the ionization balance calculation, assuming an oxygen abundance relative to hydrogen of $A_{\mathrm{O}}=6.8 \times 10^{-4}$ (Grevesse \& Sauval 1998). We will refer to this model as Model A. The XSTAR models incorporate the GAR05 cross sections for all the oxygen charge states.

\subsection{Models B, C, and D}

If a simple description of X-ray absorption by a cold medium assumes that all the spectral features are solely due to neutral oxygen photoabsorption, the observed flux can then be approximated as

$$
F(E)=F_{0} \exp \left[-N_{\mathrm{O}_{\mathrm{I}}} \sigma_{\mathrm{O}_{\mathrm{I}}}(E)\right],
$$

where $F_{0}$ is a normalization factor, $N_{\mathrm{O}_{\mathrm{I}}}$ is the oxygen column density, and $\sigma_{\mathrm{O}_{\mathrm{I}}}(E)$ is the photoabsorption cross section of $\mathrm{O} \mathrm{I}$. This is a convenient way to evaluate the relevance of the atomic data used to fit the observation. Using this assumption, we have 
Table 1

List of Models

\begin{tabular}{llccll}
\hline \hline Model & Description & Atomic Data & $\begin{array}{c}N_{\mathrm{O}} \\
\left(10^{17} \mathrm{~cm}^{-2}\right)\end{array}$ & $\chi^{2} /$ dof & \multicolumn{1}{c}{ Notes } \\
\hline A & Full XSTAR model & AKA01 $^{\mathrm{a}}+\mathrm{GAR}^{\mathrm{b}} \mathrm{b}^{\mathrm{b}}$ & $9.04 \pm 0.12$ & 2.75620 & No significant residuals \\
$\mathrm{B}$ & Atomic cross section $^{\mathrm{c}}$ & MCK98 $^{\mathrm{d}}$ & $9.25 \pm 0.07$ & 6.02484 & Large residuals near K edge \\
$\mathrm{C}$ & Atomic cross section $^{\mathrm{c}}$ & GAR05 $^{\mathrm{b}}$ & $7.94 \pm 0.22$ & 2.44650 & No significant residuals \\
D & Atomic cross section $^{\mathrm{c}}$ & GMC00 $^{\mathrm{e}}$ & $10.49 \pm 0.06$ & 2.76445 & No significant residuals \\
\hline
\end{tabular}

Notes.

a Bautista \& Kallman (2001).

b García et al. (2005).

c See Equation (1).

d McLaughlin \& Kirby (1998).

e Gorczyca \& McLaughlin (2000).

produced three additional models using Equation (1), each with a different cross section: Model B includes that computed by MCK98; Model C by GAR05; and Model D by GMC00. Note that the XSTAR model (Model A) and Model C are equivalent in the sense that they use the same atomic data although the former also includes the background due to $\mathrm{H}$ and He. These models are summarized in Table 1.

\section{RESULTS}

The models described in Section 3 are used to fit the absorption features observed in the X-ray spectrum of Sco X-1. In this respect and to determine the corresponding statistics, we use the X-ray spectral package XSPEC v12.3.0, and all the fits are carried out in the 21.0-24.5 $\AA$ spectral region. Figure 2 shows the fit using Model A, our main model, since it is the result of a self-consistent photoionization calculation and incorporates the most recent atomic cross section for $\mathrm{O}$ I by GAR05. In the upper panel, the black and gray data points belong to exposures S005 and S018, respectively. The best fit using Model A is shown with solid lines, red and blue, respectively, corresponding to the fit applied to each exposure. The spectrum displays a very strong atomic K edge which covers the 22.8-23.3 $\AA$ wavelength range. The $\mathrm{K} \alpha$ absorption resonance at $\sim 23.5 \AA$ is also one of the strongest features while the $\mathrm{K} \beta$ is much weaker but is still detectable at $\sim 22.9 \AA$. In the lower panel, we show the residuals with respect to the model in $\sigma$ units (standard deviation). The black and gray points correspond to exposures S005 and S018, respectively. Model A fits the $\mathrm{K}$ edge and the $\mathrm{K} \alpha$ absorption line successfully in the two exposures of Sco X-1. Statistics for the combined fit (i.e., for the two exposures) show a reduced chi-square of $\chi^{2} /$ dof $=2.75620$ (where dof is the number of degrees of freedom). The best-fit hydrogen column density is $N_{\mathrm{H}}=(1.33 \pm 0.02) \times 10^{21} \mathrm{~cm}^{-2}$ which corresponds to an oxygen column density of $N_{\mathrm{O}}=(9.04 \pm 0.12) \times 10^{17} \mathrm{~cm}^{-2}$.

In order to get a better grasp of the atomic data effects on the description of the observed spectra, we now fit the measurements with the simple models based on the raw photoabsorption cross section of neutral oxygen (see Equation (1)). In Figure 3 we depict the fits using Models B and C; in the upper panel, the black/gray data points are the measurements while the red/ blue curves are the models corresponding to exposures S005/ S018, respectively. The best fits using Models B and C are respectively shown with dashed and solid lines. The middle panel gives the residuals in $\sigma$ units with respect to Model $\mathrm{B}$ and the lower panel those with respect to Model C. It is important to note that, if these two models were equivalent, the dashed and solid lines with the same color would be close to each other. However, there is a clear discrepancy between the dashed lines (Model B) and the solid lines (Model C) in the region near the oxygen $\mathrm{K}$ edge $(\sim 22.5-23 \AA$ ). These differences may be appreciated in the residuals of the Model B fit shown in the middle panel. Furthermore, the residuals also indicate that Model B cannot completely fit the intensity of the $\mathrm{K} \alpha$ absorption line at $\sim 23.5 \AA$, it predicts an oxygen column density of $N_{\mathrm{O}}=$ $(9.25 \pm 0.07) \times 10^{17} \mathrm{~cm}^{-2}$, and the fit statistics give a reduced chi-square of $\chi^{2} /$ dof $=6.02484$ which is poorer than Model A. As expected, the fit using the raw cross section by GAR05 (Model C) is equivalent to that using Model A giving a reduced chi-square of $\chi^{2} /$ dof $=2.44650$. Model $\mathrm{C}$ predicts an oxygen column density of $N_{\mathrm{O}}=(7.94 \pm 0.22) \times 10^{17} \mathrm{~cm}^{-2}$ somewhat smaller than Models A and B. The differences between Models $\mathrm{A}$ and $\mathrm{C}$ may be due to the numerical interpolation used in the storage and retrieval of the cross sections by the XSTAR package.

Model B is the same as that used by de Vries \& Costantini (2009) to fit the oxygen absorption in the spectrum of Sco X-1. These authors claimed the detection of XAFS signatures in the spectra based on the relative changes in the observed flux with respect to the smooth flux predicted by the model for energies above the K edge. They also argued that the apparent shift of the observed edge with respect to the atomic model could be due to the fact that some fraction of the oxygen in the ISM is bound in solids. However, the analysis presented here shows that the large residuals found in the Model B fit are artifacts brought about by the мск98 cross section.

Finally, in Figure 4 we show a similar comparison using the GAR05 and the GMC00 cross sections (Models C and D). As before, the upper panel shows the Sco X-1 spectrum and the best-fit models. The black/gray data points are the observations while the red/blue curves are the models corresponding to exposures S005/S018, respectively. Note that Figure 4 covers the 22.5-24.0 $\AA$ wavelength range to enhance the K-edge region. The best fits using Models $\mathrm{C}$ and D are, respectively, shown with solid and dashed lines. The middle and lower panels give the residuals in $\sigma$ units for Models $\mathrm{D}$ and $\mathrm{C}$. The two models are equivalent giving similar fits $\left(\chi^{2} /\right.$ dof $=2.76445$ for Model D). This is consistent with the accord between the GMC00 and GAR05 cross sections. The oxygen column density derived from this model, $N_{\mathrm{O}}=(10.49 \pm 0.06) \times 10^{17} \mathrm{~cm}^{-2}$, is larger than those estimated by all the previous models, but consistent within the uncertainties in the parameters predicted by Models $\mathrm{A}$ and $\mathrm{C}$. The discrepancies in the predicted oxygen columns in Models $\mathrm{C}$ and $\mathrm{D}$ can be explained by those in their respective cross sections. In this small spectral region, the oxygen column depends almost entirely on the depth of the $\mathrm{K}$ edge. By comparing the values of the cross section at 22.8 

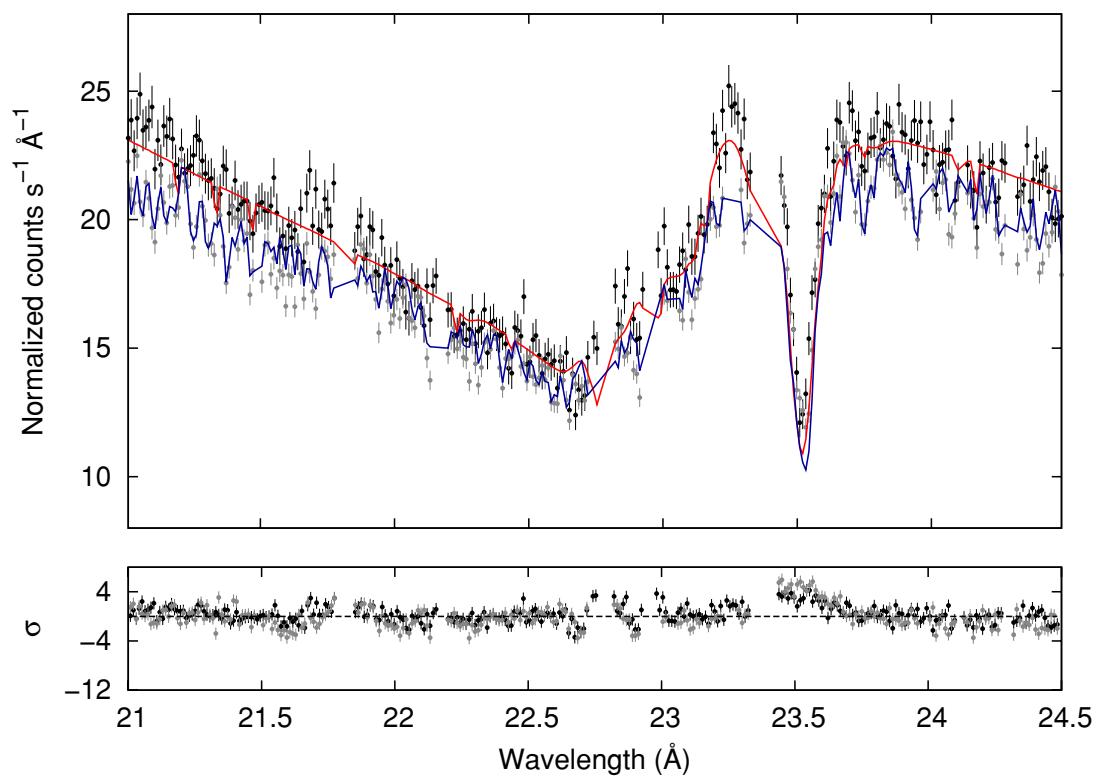

Figure 2. Sco X-1 spectrum produced by RGS1 aboard XMM-Newton covering the 21.0-24.5 ̊̊ wavelength region. In the upper panel, the black and gray data points correspond to exposures S005 and S018, respectively, while the best fits to each exposure using the XSTAR photoionization model (Model A) are respectively depicted in red and blue. The lower panel shows the residuals in $\sigma$ units in the same range with respect to the model. Black and gray points correspond to exposures S005 and S018, respectively.
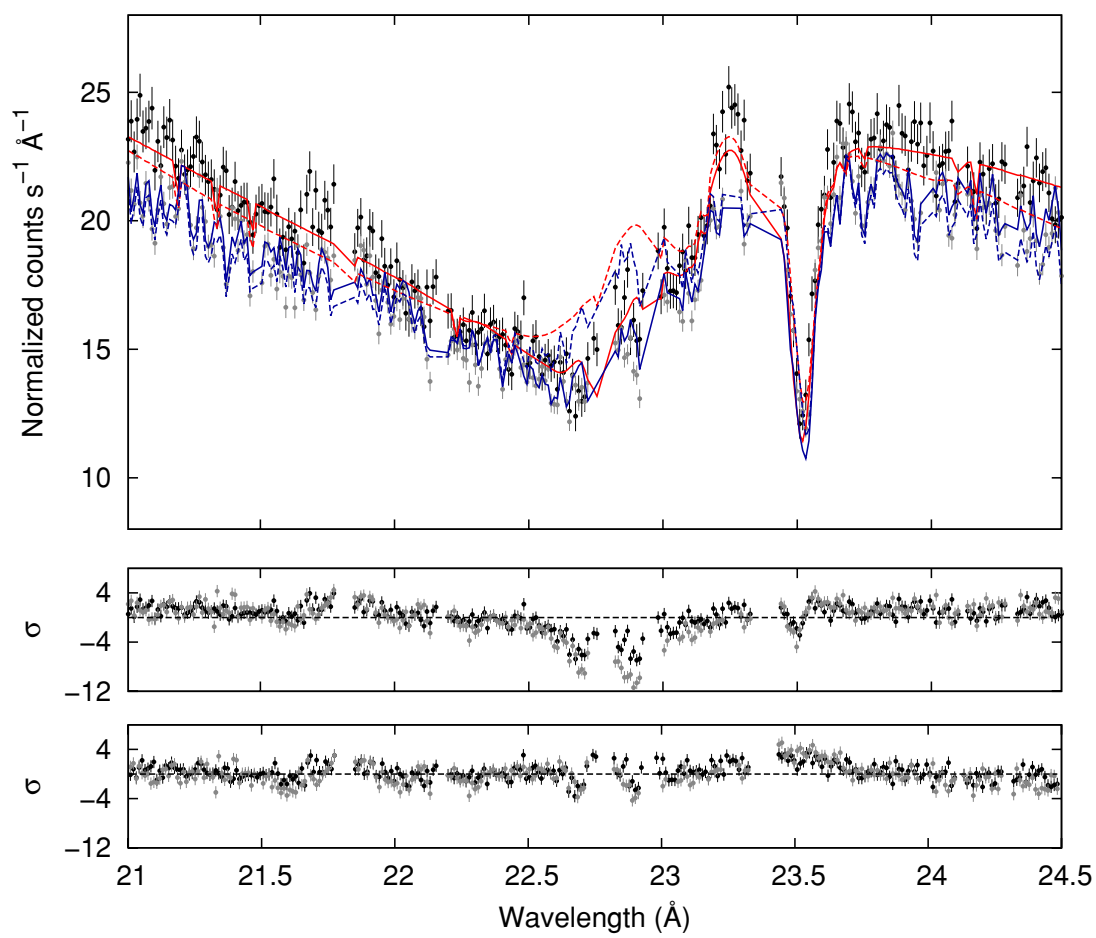

Figure 3. Spectral fit comparison using two different models. Upper panel: Sco X-1 spectrum covering the 21.0-24.5 A wavelength region. The black/gray data points are the observed data while the red/blue curves are the models corresponding to exposures S005/S018, respectively. The best fits using Models B and C are shown with the dashed and solid lines, respectively. Middle panel: residuals in $\sigma$ units with respect to Model B. Lower panel: residuals in $\sigma$ units with respect to Model C.

and $23.0 \AA$ (i.e., before and after the K edge) in Figure 1, it may be noticed that the K edge in GMC00 is weaker than in GAR05. If a feature is weaker in a model, it will require a larger column density to reproduce the observation and, therefore, explains the different column densities obtained. All the proposed models and the parameters derived from their corresponding fits are summarized in Table 1.

In all the fits presented here, we see residuals of around $\pm 2 \sigma$ distributed homogeneously along the entire spectral range considered in our analysis, consistent with the reduced chi-square close to 2 obtained in our best fit. This suggests that most of the fit errors are systematic. However, we notice significant residuals at wavelengths shorter than the $\mathrm{K} \alpha$ line. Due to a gap in the 23.33-23.44 $\AA$ wavelength range, the detection of spectral features is not possible; nonetheless, both the atomic $\mathrm{K} \alpha$ resonance due to ionized oxygen and the $1 s \rightarrow \pi^{*}$ resonance due to molecular oxygen occur at $23.35 \AA$. The former alternative is consistent with the analysis by Juett et al. (2004) 

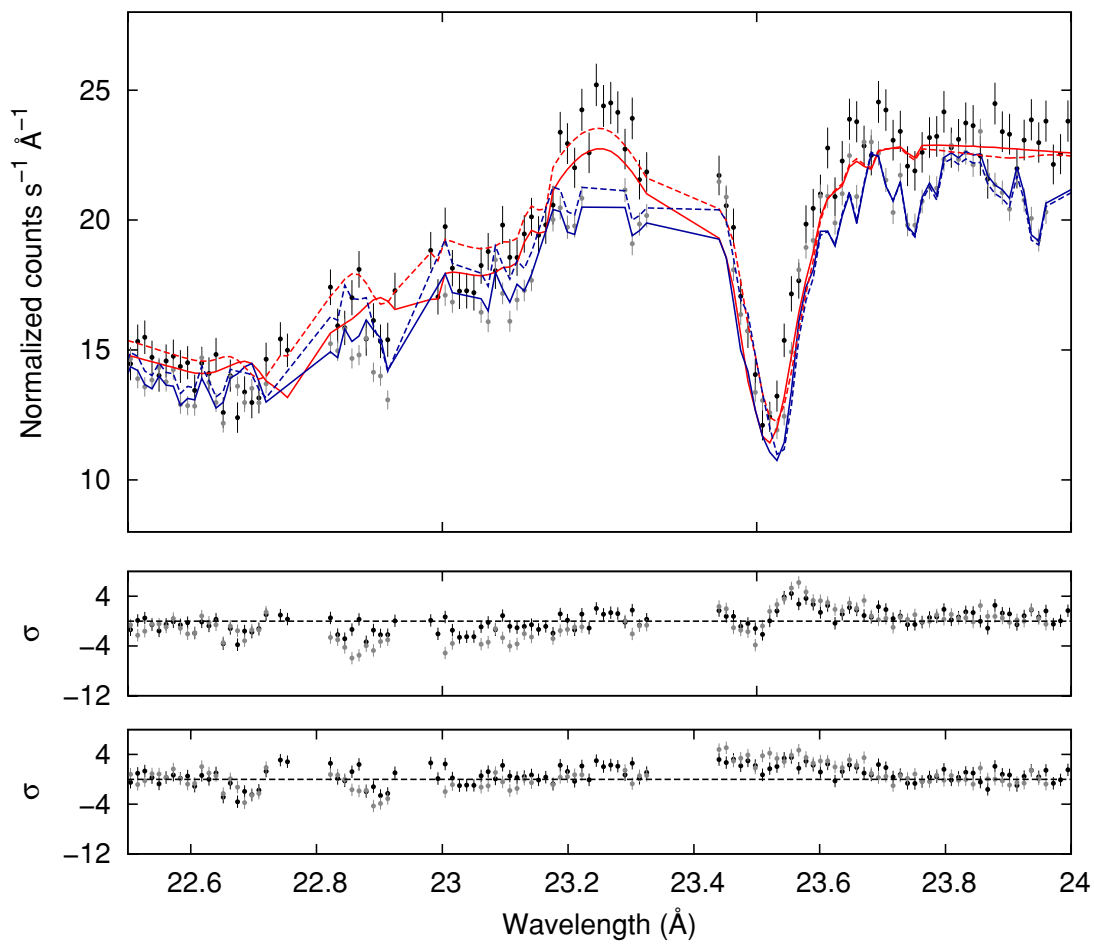

Figure 4. Spectral fit comparison using two different models. Upper panel: Sco X-1 spectrum in the 22.5-24.0 ̊ wavelength region. The black/gray data points are the observed data while the red/blue curves are the models to exposures S005/S018, respectively. The best fits using Models C and D are shown with solid and dashed lines, respectively. Middle panel: residuals in $\sigma$ units with respect to Model D. Lower panel: residuals in $\sigma$ units with respect to Model C.

where they detected $\mathrm{O}$ II absorption in the spectra of several $\mathrm{X}$-ray binaries. On the other hand, our fit using Model A predicts that absorption occurs in a mostly neutral gas with an ionization parameter of $\xi=10^{-4} \mathrm{erg} \mathrm{cm} \mathrm{s}^{-1}$ which would also suggest molecular absorption. Therefore, given the lack of data in this particular spectral range, we cannot rule out the presence of molecules in the observation in hand.

\section{CONCLUSIONS}

In the present Letter, we have shown the relevance of accurate atomic data in the modeling of the X-ray spectra from cosmic sources. In particular, we have studied the Sco X-1 spectrum produced by the RGS1 instrument on board of the XMMNewton satellite covering the 21.0-24.5 $\AA$ wavelength region. Absorption occurs when the X-rays interact with the cold gas of the ISM, the main spectral features in this region being the absorption $\mathrm{K}$ edge and $\mathrm{K} \alpha$ line from neutral oxygen. We found a good fit using a self-consistent photoionization model which includes the most recent atomic data for the oxygen isonuclear sequence by GAR05. Our fits indicate that the absorbing gas has an ionization parameter of $\xi=10^{-4} \mathrm{erg} \mathrm{cm} \mathrm{s}^{-1}$ and a hydrogen column density of $N_{\mathrm{O}} \approx(8-10) \times 10^{17} \mathrm{~cm}^{-2}$.

Simple models based on the raw atomic photoabsorption cross sections of $\mathrm{O}$ I from three different calculations were used to evaluate data sensitivity. We show that models based on the MCK98 atomic cross sections are unable to reproduce the K-edge structure in detail, while those based on GMC00 and GAR05 yield more accurate fits of the main spectral features. The fits using the most up to date models do not show evidence for absorption by anything other than atomic oxygen.

The analysis presented here indicates that the atomic data uncertainties in combination with the limited resolution of the grating spectrum make detection of molecular or solid material challenging. Although oxygen is expected to be found in molecular form or locked into solids in the ISM, the use of accurate atomic calculations to correctly account for the atomic oxygen contribution is crucial when searching for XAFS or similar features in the X-ray spectra of astronomical sources.

We thank Tom Gorczyca for providing the MCK 98 and GMC00 calculations. This work was supported by a grant from the NASA astrophysics theory program 05-ATP05-18. This research has made extensive use of the NASA Astrophysics Data System.

\section{REFERENCES}

Bautista, M. A., \& Kallman, T. R. 2001, ApJS, 134, 139

Bradshaw, C. F., Fomalont, E. B., \& Geldzahler, B. J. 1999, ApJ, 512, L121

Costantini, E., Freyberg, M. J., \& Predehl, P. 2005, A\&A, 444, 187

De Villiers, J. -P., Hawley, J. F., \& Krolik, J. H. 2003, ApJ, 599, 1238

de Vries, C. P., \& Costantini, E. 2009, A\&A, 497, 393

de Vries, C. P., den Herder, J. W., Kaastra, J. S., Paerels, F. B., den Boggende, A. J., \& Rasmussen, A. P. 2003, A\&A, 404, 959

den Herder, J. W., et al. 2001, A\&A, 365, L7

Draine, B. T. 2003, ARA\&A, 41, 241

Forrey, R. C., Woo, J. W., \& Cho, K. 1998, ApJ, 505, 236

García, J., Mendoza, C., Bautista, M. A., Gorczyca, T. W., Kallman, T. R., \& Palmeri, P. 2005, ApJS, 158, 68

Gorczyca, T. W., \& McLaughlin, B. M. 2000, J. Phys. B: At. Mol. Opt. Phys., 33, L859

Grevesse, N., \& Sauval, A. J. 1998, Space Sci. Rev., 85, 161

Juett, A. M., Schulz, N. S., \& Chakrabarty, D. 2004, ApJ, 612, 308

Juett, A. M., Schulz, N. S., Chakrabarty, D., \& Gorczyca, T. W. 2006, ApJ, 648, 1066

Kaastra, J. S., de Vries, C. P., Costantini, E., \& den Herder, J. W. A. 2009, A\&A, 497, 291

Lee, J. C. 2011, Space Sci. Rev., in press

Lee, J. C., Ogle, P. M., Canizares, C. R., Marshall, H. L., Schulz, N. S., Morales, R., Fabian, A. C., \& Iwasawa, K. 2001, ApJ, 554, L13

Lee, J. C., \& Ravel, B. 2005, ApJ, 622, 970

Lee, J. C., Reynolds, C. S., Remillard, R., Schulz, N. S., Blackman, E. G., \& Fabian, A. C. 2002, ApJ, 567, 1102 
Lee, J. C., Xiang, J., Ravel, B., Kortright, J., \& Flanagan, K. 2009, ApJ, 702, 970

McLaughlin, B. M., \& Kirby, K. P. 1998, J. Phys. B: At. Mol. Opt. Phys., 31, 4991

Paerels, F., et al. 2001, ApJ, 546, 338

Pinto, C., Kaastra, J. S., Costantini, E., \& Verbunt, F. 2010, A\&A, 521, A79

Schulz, N. S., Cui, W., Canizares, C. R., Marshall, H. L., Lee, J. C., Miller, J. M., \& Lewin, W. H. G. 2002, ApJ, 565, 1141

Stolte, W. C., et al. 1997, J. Phys. B: At. Mol. Opt. Phys., 30, 4489
Takei, Y., Fujimoto, R., Mitsuda, K., \& Onaka, T. 2002, ApJ, 581, 307

Tarter, C. B., Tucker, W. H., \& Salpeter, E. E. 1969, ApJ, 156, 943

Turner, A. K., Fabian, A. C., Lee, J. C., \& Vaughan, S. 2004, MNRAS, 353, 319

Ueda, Y., Mitsuda, K., Murakami, H., \& Matsushita, K. 2005, ApJ, 620, 274

Whittet, D. C. B. 2003, Dust in the Galactic Environment (2nd ed.; Bristol: Institute of Physics Publishing)

Woo, J. W. 1995, ApJ, 447, L129

Woo, J. W., Forrey, R. C., \& Cho, K. 1997, ApJ, 477, 235 